\title{
Studies on Different Genotypes of Gaillardia (Gaillardia pulchella L.) for Quantitative and Qualitative Performance
}

\author{
Nagesh Gawade*, S.G. Bhalekar, Pushpanjali Bhosale, \\ S.M. Katwate and Vaibhav Wadekar
}

Department of Horticulture, College of Agriculture, Pune-05, MPKV, Rahuri-413722, India

*Corresponding author

\section{A B S T R A C T}

\section{Keywords}

Gaillardia (Gaillardia pulchella $\mathrm{L}$. genotypes,

Quantitative and qualitative characters

\section{Article Info}

Accepted:

10 February 2018

Available Online:

10 March 2018
The present investigation was carried out during the year 2015-2016 at Modibaug garden of Horticulture Section, College of Agriculture, Pune. The experiment was laid out in Randomized Block Design with two replications and forty genotypes. The genotype MG$10-2$ recorded significantly highest plant height $(80.52 \mathrm{~cm}),(99.50 \mathrm{~cm})$ and $(102.86 \mathrm{~cm})$ at 90,120 and 150 days after transplanting respectively. The genotype MG-9-1 $(90.28 \mathrm{~cm})$ recorded significantly maximum plant spread at (E-W) direction and the genotype MG-3-2 $(80.34 \mathrm{~cm})$ at $(\mathrm{N}-\mathrm{S})$ direction at 90 DAP. Significantly maximum number of primary and secondary branches per plant was recorded in the genotypes MG-7-2 (21.48) and MG-10-2 (58.50) respectively. The genotype MG-9-1 showed significantly early initiation of flowering (41.23 days) after transplanting. The genotype MG-9-1 showed early 50 percent flowering (64.38 days) but the treatment differences were not significant. The genotype MG-7-2 recorded significantly maximum flower diameter $(6.93 \mathrm{~cm})$ followed by the genotype MG-9-1 $(6.82 \mathrm{~cm})$ and both genotypes are on par with each other. Significantly more number of ray florets per flower was noticed in the genotypes MG-5-1 (148.40), MG-5-2 (148.20) and MG-9-1 (148.05). The genotype MG-2-2 recorded significantly maximum flower stalk length $(17.62 \mathrm{~cm})$. The flower stalk thickness of different genotypes of gaillardia ranged from 2.8 to $3.1 \mathrm{~mm}$ but the treatment differences were nonsignificant for this character. Significantly more number of flowers per plant was recorded in the genotype MG-2-2 (288.35) followed by the genotypes MG-7-1 (263.23) and MG-91 (242.43). The genotypes MG-9-1 and MG-7-2 recorded significantly maximum weight of 100 flowers $(333 \mathrm{~g})$. The weight of flowers per plant, yield of flowers per plot and yield of flowers per hectare were recorded significantly highest in MG-9-1 (631.00 g, $12.02 \mathrm{~kg}$ and 33.30 tonne) respectively. The Aureolin flower colour, Lemon Yellow flower colour, Buttercup Yellow flower colour and Indian Yellow flower colour were observed in two, twelve, twelve, fourteen genotypes of gaillardia respectively The shape of petals of genotype MG-4-2, MG-5-3, MG-6-2, MG-7-2, MG-10-1 and MG-10-5 of gaillardia had narrow with blunt tip and remaining genotypes had shown narrow with pointed tip. The genotype MG-6-1 and MG-14-1 had dense pubescence. The genotypes MG-2-1, MG-3-1, MG-5-4, MG-7-1 and MG-10-2 had medium pubescence. The remaining 33 genotypes had comparatively low pubescence. 


\section{Introduction}

Gaillardia (Gaillardia pulchella L.) is one of the important flower crop of Maharashtra grown on commercial scale as loose flower. In India, Gaillardia is grown all the year around. The generic name Gaillardia was proposed in honour of Mr. M. Gaillard, a French patron of botany, who cultivated it first. The Central and Western United states are considered to be its origin.

It is popularly known as 'blanket flower' and belongs to family asteraceae. It is one of the hardiest annual can be grown in a wide range of tropical to temperate climate. This is substitute flower crop for chrysanthemum and China aster (Bose et al., 2003).

There are about twenty-eight species reported in the genus Gaillardia, but only two of them viz. Gaillardia pulchella (annual) and Gaillardia aristica (perennial) are in cultivation. Gaillardia pulchella is diploid $(2 \mathrm{n}=36)$ as reported by Morinaga et al., (1929).

Gaillardia are grown in herbaceous borders, beds and are also suitable for cut flowers. Besides, its utility in landscape Gaillardia pulchella is useful in reducing erosion in coastal dune areas (Carig, 1977). Gaillardia aristata is suitable for dryland and requires low maintenance, (Cox and Klett, 1984; Robinson and Schultz, 1995). Panchaude (1990) reported the nematicidal property of gaillardia, when grown as catch crop and green manure.

The commercial importance and popularity of the crop, there is a lot of scope for breeding of new varieties with higher yield and superior quality of flowers. With a view, to find out superior genotypes having high yield and good quality flowers, the present investigation was planned at Pune.

\section{Materials and Methods}

The present investigation was initiated in Modibaug Garden of Horticulture Section, College of Agriculture, Pune during 20152016. Pune is situated in the mid-west Maharashtra at an altitude of $559 \mathrm{~m}$, above MSL. It is located in tropical region at $18.32^{\circ}$ $\mathrm{N}$ latitude and $73.51^{\circ} \mathrm{E}$ longitudes. The average annual rainfall in this area is 650-750 $\mathrm{mm}$. The average maximum and minimum temperature recorded during the period of experiment was $39.1^{\circ} \mathrm{C}$ and $14.4^{\circ} \mathrm{C}$, respectively. The relative humidity during crop growth period ranged between $45 \%$ and $85 \%$.

The material for the present investigation comprised of 40 pure lines of individual selfed plant progenies having diversity for the growth and yield characters is being used for further study. The breeding programme is continue for getting uniformity and high flower yield in gaillardia. The nomenclature of these genotypes is given below in Table 1 . The experiment was laid out in a Randomized Block Design (RBD) with two replications and 40 treatments (Table 1) with spacing $60 \mathrm{~cm} \times 30 \mathrm{~cm}$.

During the study, the observations of growth, flowering and yield characters were recorded at regular interval throughout the period of the gaillardia crop. Five plants from each treatment plot were randomly selected, labeled and used for recording observations. For the growth characters, the plant height was recorded in centimeters at 90, 120 and 150 DAT (days after transplanting) from each treatment plot. Plant spread (E-W and N-S) and total numbers of primary and secondary branches were counted at 90 DAT from each treatment plot. To assess performance of various genotypes on flowering behavior and flowering characters, the observations were recorded on days to initiation of flowering, 
days to $50 \%$ flowering, flower diameter $(\mathrm{cm})$, number of ray florets per flower, flower stalk length and flower stalk thickness. Also, observations on yield characters such as, number of flowers per plant, weight of flowers per plant $(\mathrm{g})$, weight of 100 flowers $(\mathrm{g})$, yield of flowers per plot $(\mathrm{kg})$, yield of flowers per hectare (tonne) were recorded from each net plot. The colour of the fully opened flower was recorded before they started fading by comparing their colour with colour shades mentioned in Royal Horticulture Society colour chart. From the observational plants from each treatment plot, the shape of petal and pubescence were recorded.

\section{Results and Discussion}

From the data, it can be revealed that the height of the plants differed significantly in different genotypes of gaillardia. The genotype MG-10-2 recorded significantly highest plant height $(80.52 \mathrm{~cm}),(99.50 \mathrm{~cm})$ and $(102.86 \mathrm{~cm})$ at 90,120 and 150 days after transplanting respectively. Among the forty genotypes ten genotypes viz., MG-3-2, MG-91, MG-4-1, MH-9-4, MG-9-2, MG-2-2, MG5-5, MG-7-2 and MG-6-2 were at par with genotype $\mathrm{MG}-10-2$. The present findings in respect of plant height, in general are in agreement with those reported by Agale (2012) in gaillardia, Palai et al., (1999) in chrysanthemum, Swaroop et al., (2004) in China aster and Bhati and Chitkara (1989) in marigold. The plant spread differed significantly in different genotypes of gaillardia under study when measured at 90 days after transplanting. The plant spread at (E-W) direction was significantly maximum in the genotype MG-9-1 (90.28 cm) and minimum plant spread was recorded in the genotype MG-7-3 (48.39 cm). The plant spread at $(\mathrm{N}-\mathrm{S})$ direction was the highest in the genotype MG-3-2 (80.34 cm). The genotype MG-9-1 $(76.48 \mathrm{~cm})$ was at par with the genotype MG-3-2. The minimum plant spread was noticed by the genotype MG-14-1 $(39.61 \mathrm{~cm})$. The difference in plant spread is may be due to varietal trait and similar results were obtained by Agale (2012) in gaillardia. The number of primary branches per plant was significantly maximum in the genotype MG-72 (21.48) and minimum number of primary branches per plant was recorded by the genotype MG-3-4 (7.95). The number of secondary branches per plant was significantly maximum in the genotype MG-9-1 (58.50) and minimum number of secondary branches per plant was recorded by the genotype MG-61 (25.07). The variation in number of branches per plant may be due to genetic trait of the genotype. These findings were confounded by Agale (2012) in gaillardia. In his study the primary branches were ranged from 17.40 to $20.47 \mathrm{~cm}$ and these findings are similar to Kale (2002) in gaillardia.

Significantly earlier flowering was observed in the genotype MG-9-1 (41.23 days). The genotypes MG-2-2 (47.16 days), MG-6-2 (47.31 days), MG-5-3 (47.34 days) and MG-43 (49.61 days) were at par with the genotype MG-9-1. The genotype MG-13-1 required significantly maximum days for commencement of flowering (62.84 days). Similar observations have been reported by Zosiamliana et al., (2011) and Mohanty et al., (2003) in marigold. Early or late flowering behavior is a genotypic characteristic with support of genetic base as well as physiological characteristics. It is seen from the data that there was no any significant difference between different genotypes of gaillardia for 50 per cent flowering. The data showed that the days required for 50 per cent flowering were lowest in the genotype MG-91 (64.38 days) while highest in the genotype MG-13-1 (78.94 days). These findings were confounded by Agale (2012) in gaillardia and Bhuyar et al., (2004) in gerbera. Diameter of flower is an important character regarding the flower quality and in the present study it 
appeared to be the genotype characteristic. The significantly maximum flower diameter $(6.93 \mathrm{~cm})$ was recorded in the genotype MG7-2 followed by the genotype MG-9-1 (6.82 $\mathrm{cm})$ which was at par with the genotype MG7-2. The minimum flower diameter was recorded by the genotype MG-4-3 $(4.25 \mathrm{~cm})$. This variation may be due to differences in the genetic makeup of the genotypes. The present findings regarding flower size are in close conformity with observations recorded by Agale (2012) and observed that the significantly maximum diameter of flower was produced by MG-6 $(7.27 \mathrm{~cm})$ over the rest of the genotypes. The remaining genotypes flower diameter was ranged from 4.67 to $6.47 \mathrm{~cm}$. Similarly, Kale (2002) studied on variability, heritability, correlation and path analysis in gaillardia and reported that maximum flower diameter was recorded in $\mathrm{KRC}-1 \quad(6.03 \mathrm{~cm})$ while, minimum in Bangalore Local $(4.73 \mathrm{~cm})$. The significantly maximum number of ray florets per flower was recorded by the genotype MG-5-1 (148.40) and minimum in the genotype MG-81 (99.30). The variation in number of ray florets per flower may be due to genetic trait. These findings are also in accordance with Swaroop et al., (2008) evaluated chrysanthemum and reported that the highest number of ray florets were recorded in Thai Chin Queen (276.33) while minimum in Snowball (89.33) in 2005. Whereas maximum ray florets were recorded in variety Flirt (308.66) and minimum in Snowball (90.00) in 2006. Significantly maximum flower stalk length was noticed by the genotype MG-2-2 $(17.62 \mathrm{~cm})$ and about 14 genotypes were at par with this genotype. The minimum flower stalk length was recorded by the genotype MG-1-4 $(7.73 \mathrm{~cm})$. It is seen from the data that treatment differences were non-significant for flower stalk thickness. The maximum flower stalk thickness was recorded by genotypes MG-1-3 and MG-5-2 (3.1 mm each). Similar results have been reported by
Agale (2012) in gaillardia and observed that there were no significant differences among the genotypes of gaillardia. He noticed the maximum flower stalk thickness $(3.0 \mathrm{~mm})$ was recorded in MG-1, MG-5, MG-6, MG-7, MG10, and MG-13.

Significantly maximum number of flowers per plant was obtained by the genotype MG-2-2 (288.35). The genotypes MG-7-1 (263.23), MG-9-1 (242.43), MG-6-2 (228.71), local check (226.91) and MG-8-2 (221.10) were at with genotype MG-2-2. The minimum number of flowers per plant was recorded by the genotype MG-5-4 (102.05). This finding was supported by Agale (2012) in gaillardia and Palai (2009) in chrysanthemum. The data indicate that yield of flower per plant varied significantly with different genotypes due to flower diameter, number of ray florets per plant and number of flowers per plant. Significantly maximum flower yield per plant was obtained in the genotype MG9-1 (631 g) followed by the genotypes MG-7-2 (600.76 g), MG-6-2 (588.31 g), MG-2-2 (563.13 g), MG5-5 (535.69 g) and MG-2-1 (531.09 g) were at par with genotype MG-9-1. The minimum flower yield per plant was recorded by the genotype MG-4-3 (317.74 g). These findings were confounded by Agale (2012) in gaillardia and Jagtap (2013) in china aster. In the present study, the maximum weight of flowers was recorded significantly by MG-7-2 and MG-9-1 (333 g each) genotypes and minimum weight of flower was recorded by the genotype MG4-3 (179 g). These might be due to maximum flower diameter and maximum number of ray florets. Similar findings also reported by Agale (2012) in gaillardia and observed that the maximum weight of the flowers was recorded significantly by MG-6 (420 g). The other genotypes ranged from 270 to $380 \mathrm{~g}$. The genotype MG-9-1 (12.02 kg) recorded significantly higher yield of flowers per plot. Minimum flower yield $(5.96 \mathrm{~kg})$ per plot was recorded by the genotype MG-4-3. 
Table.1 Treatment details

\begin{tabular}{|c|c|c|c|}
\hline Treatments & $\begin{array}{l}\text { Name of } \\
\text { genotypes }\end{array}$ & Treatments & $\begin{array}{l}\text { Name of } \\
\text { genotypes }\end{array}$ \\
\hline $\mathbf{T}_{1}$ & MG-1-3 & $\mathrm{T}_{21}$ & MG-7-2 \\
\hline $\mathbf{T}_{2}$ & MG-1-4 & $\mathrm{T}_{22}$ & MG-7-3 \\
\hline $\mathbf{T}_{3}$ & MG-2-1 & $\mathrm{T}_{23}$ & MG-8-1 \\
\hline $\mathbf{T}_{4}$ & MG-2-2 & $\mathrm{T}_{24}$ & MG-8-2 \\
\hline $\mathbf{T}_{5}$ & MG-3-1 & $\mathrm{T}_{25}$ & MG-9-1 \\
\hline$T_{6}$ & MG-3-2 & $\mathrm{T}_{26}$ & MG-9-2 \\
\hline $\mathbf{T}_{7}$ & MG-3-3 & $\mathrm{T}_{27}$ & MG-9-3 \\
\hline$T_{8}$ & MG-3-4 & $\mathrm{T}_{28}$ & MG-9-4 \\
\hline$T_{9}$ & MG-4-1 & $\mathrm{T}_{29}$ & MG-10-1 \\
\hline$T_{10}$ & MG-4-2 & $\mathrm{T}_{30}$ & MG-10-2 \\
\hline$T_{11}$ & MG-4-3 & $\mathrm{T}_{31}$ & MG-10-3 \\
\hline$T_{12}$ & MG-4-4 & $\mathrm{T}_{32}$ & MG-10-4 \\
\hline $\mathbf{T}_{13}$ & MG-5-1 & $\mathrm{T}_{33}$ & MG-10-5 \\
\hline$T_{14}$ & MG-5-2 & $\mathrm{T}_{34}$ & MG-11-1 \\
\hline$T_{15}$ & MG-5-3 & $\mathrm{T}_{35}$ & MG-12-1 \\
\hline$T_{16}$ & MG-5-4 & $\mathrm{T}_{36}$ & MG-12-2 \\
\hline $\mathbf{T}_{17}$ & MG-5-5 & $\mathrm{T}_{37}$ & MG-12-3 \\
\hline$T_{18}$ & MG-6-1 & $\mathrm{T}_{38}$ & MG-13-1 \\
\hline$T_{19}$ & MG-6-2 & $\mathrm{T}_{39}$ & MG-14-1 \\
\hline $\mathbf{T}_{20}$ & MG-7-1 & $\mathrm{T}_{40}$ & Local (C) \\
\hline
\end{tabular}

Table.2 Growth, flowering and yield characters

\begin{tabular}{|l|l|}
\hline Genotypes \\
\hline MG-1-3 \\
\hline MG-1-4 \\
\hline MG-2-1 \\
\hline MG-2-2 \\
\hline MG-3-1 \\
\hline MG-3-2 \\
\hline MG-3-3 \\
\hline MG-3-4 \\
\hline MG-4-1 \\
\hline MG-4-2 \\
\hline MG-4-3 \\
\hline MG-4-4 \\
\hline MG-5-1 \\
\hline MG-5-2 \\
\hline MG-5-3 \\
\hline MG-5-4 \\
\hline MG-5-5 \\
\hline MG-6-1 \\
\hline MG-6-2 \\
\hline MG-7-1 \\
\hline MG-7-2 \\
\hline
\end{tabular}

\begin{tabular}{|c|c|c|}
\hline \multicolumn{3}{|c|}{ Mean plant height $(\mathbf{c m})$} \\
\hline 90 DAT & 120 DAT & 150 DAT \\
\hline 70.03 & 87.96 & 90.96 \\
\hline 70.14 & 80.81 & 84.6 \\
\hline 64.42 & 83.13 & 86.65 \\
\hline 74.21 & 91.83 & 94.72 \\
\hline 72.1 & 92.16 & 92.59 \\
\hline 79.22 & 98.81 & 102.49 \\
\hline 68.81 & 88.99 & 92.89 \\
\hline 66.8 & 84.66 & 88.05 \\
\hline 76.01 & 94.27 & 97.57 \\
\hline 64 & 82.65 & 86.89 \\
\hline 71.81 & 87.96 & 91.91 \\
\hline 66.22 & 86.98 & 91 \\
\hline 72.04 & 89.48 & 92.94 \\
\hline 67.22 & 84.16 & 87.57 \\
\hline 70.53 & 89.83 & 93.92 \\
\hline 67.92 & 88.66 & 92.08 \\
\hline 72.67 & 89.12 & 96.02 \\
\hline 64.5 & 84.33 & 88.08 \\
\hline 72.17 & 94.16 & 97.37 \\
\hline 66 & 86.16 & 89.18 \\
\hline 72.57 & 91.5 & 95.69 \\
\hline & & \\
\hline
\end{tabular}

\begin{tabular}{|c|c|}
\hline \multicolumn{2}{|c|}{ Mean plant spread } \\
\hline E-W & N-S \\
\hline 61.39 & 47.36 \\
\hline 52.49 & 62.13 \\
\hline 69.33 & 66.42 \\
\hline 54.47 & 59.41 \\
\hline 60.05 & 58.74 \\
\hline 59.72 & 80.34 \\
\hline 58.91 & 50.03 \\
\hline 70.16 & 61.28 \\
\hline 73.37 & 57.65 \\
\hline 71.66 & 61.43 \\
\hline 69.68 & 60.18 \\
\hline 55.08 & 48.53 \\
\hline 55.61 & 56.19 \\
\hline 76.43 & 65.34 \\
\hline 49.68 & 53.18 \\
\hline 73.29 & 56.4 \\
\hline 57.29 & 55.19 \\
\hline 59.18 & 53.06 \\
\hline 75.64 & 55.16 \\
\hline 53.24 & 52.91 \\
\hline 65.92 & 55.27 \\
\hline
\end{tabular}

\begin{tabular}{|c|c|c|c|}
\hline $\begin{array}{c}\text { Number of branches } \\
\text { per plant }\end{array}$ & $\begin{array}{c}\text { Days to initiation of } \\
\text { flowering }\end{array}$ & $\begin{array}{c}\text { Days to 50\% } \\
\text { flowering }\end{array}$ \\
\hline Pri. & Sec. & (days) & (days) \\
\hline 15.66 & 40.25 & 50.51 & 67.58 \\
\hline 13.23 & 40.09 & 51.26 & 65.49 \\
\hline 21.34 & 50.52 & 52.67 & 66.53 \\
\hline 19.65 & 43.38 & 47.16 & 67.4 \\
\hline 8.47 & 41.82 & 57.98 & 73.1 \\
\hline 10.63 & 34.06 & 50.64 & 66.59 \\
\hline 11.2 & 33.51 & 54.63 & 71.43 \\
\hline 7.95 & 26.82 & 56.34 & 73.68 \\
\hline 8.29 & 26.33 & 57.91 & 73.46 \\
\hline 12.28 & 31.96 & 55.24 & 70.56 \\
\hline 12.52 & 31.93 & 49.61 & 67.84 \\
\hline 12.3 & 33.13 & 57.91 & 73.59 \\
\hline 11.34 & 32.28 & 58.81 & 76.41 \\
\hline 16.45 & 39.98 & 56.67 & 73.62 \\
\hline 21.24 & 53.21 & 47.34 & 65.82 \\
\hline 16.71 & 41.4 & 54.68 & 69.35 \\
\hline 14.26 & 28.61 & 53.64 & 69.46 \\
\hline 11.02 & 25.07 & 53.02 & 69.48 \\
\hline 19.47 & 52.78 & 47.31 & 64.57 \\
\hline 13.24 & 30.68 & 51.03 & 68.52 \\
\hline 21.48 & 56.45 & 50.61 & 66.15 \\
\hline
\end{tabular}


Int.J.Curr.Microbiol.App.Sci (2018) 7(3): 1030-1039

\begin{tabular}{|c|c|c|c|c|c|c|c|c|c|}
\hline \multirow[t]{2}{*}{ Genotypes } & \multicolumn{3}{|c|}{ Mean plant height $(\mathrm{cm})$} & \multicolumn{2}{|c|}{ Mean plant spread } & \multicolumn{2}{|c|}{$\begin{array}{c}\text { Number of branches } \\
\text { per plant }\end{array}$} & \multirow{2}{*}{$\begin{array}{c}\text { Days to } \\
\text { initiation of } \\
\text { flowering } \\
\text { (days) }\end{array}$} & \multirow{2}{*}{$\begin{array}{c}\text { Days to } \\
50 \% \\
\text { flowering } \\
\text { (days) }\end{array}$} \\
\hline & 90 DAT & 120 DAT & 150 DAT & $\mathbf{E}-\mathbf{W}$ & $\mathbf{N}-\mathrm{S}$ & Pri. & Sec. & & \\
\hline MG-7-3 & 68.88 & 87.92 & 91.31 & 48.39 & 51.42 & 13.04 & 35.04 & 54.63 & 71.04 \\
\hline MG-8-1 & 58.37 & 79.69 & 83.46 & 63.35 & 55.49 & 16.46 & 33.51 & 56.48 & 71.59 \\
\hline MG-8-2 & 64.92 & 82.73 & 85.54 & 48.53 & 52.17 & 20.08 & 52.53 & 55.38 & 72.58 \\
\hline MG-9-1 & 76.98 & 96.63 & 101.45 & 90.28 & 76.48 & 20.08 & 58.5 & 41.23 & 64.38 \\
\hline MG-9-2 & 75.22 & 92.83 & 96.25 & 57.16 & 49.68 & 13.49 & 30.89 & 57.28 & 73.48 \\
\hline MG-9-3 & 76.17 & 95.83 & 100.01 & 73.62 & 60.79 & 15.32 & 37.79 & 52.69 & 68.72 \\
\hline MG-9-4 & 75.54 & 95.81 & 101.05 & 58.38 & 54.28 & 14.53 & 31.57 & 52.73 & 68.64 \\
\hline MG-10-1 & 70.49 & 89.66 & 93.38 & 79.96 & 59.13 & 15.49 & 39.32 & 55.84 & 71.49 \\
\hline MG-10-2 & 80.52 & 99.5 & 102.86 & 75.82 & 69.58 & 19.38 & 53.49 & 52.61 & 68.38 \\
\hline MG-10-3 & 66.06 & 85.46 & 89.00 & 62.38 & 61.29 & 10.28 & 32.03 & 54.83 & 70.42 \\
\hline MG-10-4 & 57.02 & 75.49 & 79.39 & 81.04 & 70.46 & 18.43 & 46.72 & 55.94 & 71.09 \\
\hline MG-10-5 & 70.65 & 90.5 & 94.51 & 69.29 & 48.95 & 15.29 & 40.81 & 51.62 & 68.35 \\
\hline MG-11-1 & 66.09 & 84.33 & 87.82 & 53.62 & 48.09 & 9.56 & 27.35 & 57.71 & 73.64 \\
\hline MG-12-1 & 72.01 & 89.01 & 92.9 & 67.39 & 57.86 & 19.76 & 46.56 & 60.5 & 77.14 \\
\hline MG-12-2 & 70.18 & 88.66 & 91.94 & 70.18 & 56.94 & 12.52 & 30.46 & 61.37 & 78.67 \\
\hline MG-12-3 & 70.67 & 90.99 & 94.8 & 65.28 & 55.82 & 12.72 & 31.88 & 58.91 & 73.61 \\
\hline MG-13-1 & 65.22 & 85.66 & 89.47 & 61.29 & 53.41 & 13.4 & 30.01 & 62.84 & 78.94 \\
\hline MG-14-1 & 71.46 & 87.83 & 91.71 & 57.48 & 39.61 & 16.79 & 44.87 & 52.67 & 68.51 \\
\hline Local (C) & 71.51 & 89.5 & 93.43 & 51.92 & 43.91 & 16.18 & 44.4 & 51.38 & 68.36 \\
\hline SEm \pm & 2.94 & 2.96 & 3.05 & 3.54 & 3.25 & 2.69 & 4.96 & 3.14 & 3.62 \\
\hline CD at $5 \%$ & 8.4 & 8.48 & 8.72 & 10.13 & 9.31 & 7.71 & 14.18 & 8.99 & NS \\
\hline
\end{tabular}

\begin{tabular}{|c|c|c|c|c|c|c|c|c|c|}
\hline \multirow[t]{2}{*}{ Genotypes } & $\begin{array}{l}\text { Mean } \\
\text { flower } \\
\text { dia. }\end{array}$ & \multirow{2}{*}{$\begin{array}{c}\text { Mean } \\
\text { number of } \\
\text { ray florets / } \\
\text { flower }\end{array}$} & \multirow{2}{*}{$\begin{array}{c}\text { Flower } \\
\text { stalk } \\
\text { length } \\
(\mathrm{cm})\end{array}$} & \multirow{2}{*}{$\begin{array}{c}\begin{array}{c}\text { Flower } \\
\text { stalk } \\
\text { thickness }\end{array} \\
(\mathbf{m m})\end{array}$} & \multirow[t]{2}{*}{$\begin{array}{c}\text { No. of } \\
\text { flowers/ } \\
\text { plant }\end{array}$} & \multirow{2}{*}{$\begin{array}{c}\begin{array}{c}\text { Weight of } \\
\text { flowers/ } \\
\text { plant }\end{array} \\
\text { (g) }\end{array}$} & \multirow{2}{*}{$\begin{array}{c}\begin{array}{c}\text { Weight } \\
\text { of } 100 \\
\text { flowers }\end{array} \\
(g)\end{array}$} & \multirow{2}{*}{$\begin{array}{c}\text { Yield of } \\
\text { flowers/ } \\
\text { plot } \\
\text { (kg) }\end{array}$} & \multirow{2}{*}{$\begin{array}{c}\text { Yield of } \\
\text { flowers/ } \\
\text { ha } \\
\text { (tonne) }\end{array}$} \\
\hline & $(\mathbf{c m})$ & & & & & & & & \\
\hline MG-1-3 & 5.26 & 140.2 & & 3.1 & 211.17 & 453.74 & 201 & 8.48 & 23.48 \\
\hline MG-1-4 & 5.77 & 141.6 & 7.73 & 3.0 & 210.47 & 442.57 & 202 & 8.45 & 23.41 \\
\hline MG-2-1 & 5.71 & 139.8 & 14.99 & 2.8 & 196.64 & 531.09 & 255 & 10.03 & 27.76 \\
\hline MG-2-2 & 6.01 & 139.5 & 17.62 & 2.9 & 288.35 & 563.13 & 186 & 10.67 & 29.54 \\
\hline MG-3-1 & 5.83 & 146.48 & 16.31 & 2.8 & 201.31 & 427.4 & 203 & 8.15 & 22.57 \\
\hline MG-3-2 & 5 & 146.4 & 13.33 & 2.8 & 209.51 & 491.74 & 227 & 9.24 & 25.58 \\
\hline MG-3-3 & 5.58 & 144.2 & 17.53 & 2.9 & 139.33 & 351.77 & 239 & 6.64 & 18.38 \\
\hline MG-3-4 & 5.2 & 144.2 & 14.97 & 2.8 & 183.58 & 415.04 & 216 & 7.9 & 21.89 \\
\hline MG-4-1 & 5.49 & 143.9 & 15.63 & 2.8 & 169.65 & 389.86 & 223 & 7.4 & 20.49 \\
\hline MG-4-2 & 5.14 & 141.6 & 14.88 & 2.9 & 155.49 & 374.15 & 229 & 7.09 & 19.62 \\
\hline MG-4-3 & 4.25 & 122.5 & 16.82 & 2.9 & 205.92 & 317.74 & 179 & 5.96 & 16.5 \\
\hline MG-4-4 & 5.38 & 141.25 & 16.63 & 2.8 & 126.81 & 358.9 & 271 & 6.78 & 18.78 \\
\hline MG-5-1 & 5.99 & 148.4 & 15.81 & 3.0 & 160.93 & 366.39 & 215 & 6.93 & 19.19 \\
\hline MG-5-2 & 5.85 & 148.2 & 14.41 & 3.1 & 213.43 & 429.93 & 193 & 8.2 & 22.71 \\
\hline MG-5-3 & 5.86 & 143.4 & 14.98 & 3.0 & 194.67 & 407.19 & 200 & 7.75 & 21.45 \\
\hline MG-5-4 & 5.69 & 144.2 & 14.37 & 3.0 & 102.05 & 358.9 & 264 & 6.78 & 18.78 \\
\hline MG-5-5 & 6.13 & 147 & 15.98 & 2.9 & 203 & 535.69 & 249 & 10.12 & 28.02 \\
\hline MG-6-1 & 5.93 & 144.2 & 10.79 & 2.9 & 187.67 & 440.49 & 227 & 8.41 & 23.3 \\
\hline MG-6-2 & 5.87 & 145.4 & 15.25 & 3.0 & 228.71 & 588.31 & 245 & 11.17 & 30.93 \\
\hline MG-7-1 & 5.93 & 143.4 & 13.32 & 2.9 & 263.23 & 506.9 & 186 & 9.54 & 26.42 \\
\hline MG-7-2 & 6.93 & 141.6 & 16.58 & 2.8 & 175.25 & 600.76 & 333 & 11.42 & 31.62 \\
\hline
\end{tabular}




\begin{tabular}{|c|c|c|c|c|c|c|c|c|c|}
\hline \multirow[t]{2}{*}{ Genotypes } & $\begin{array}{l}\text { Mean } \\
\text { flower } \\
\text { dia. }\end{array}$ & \multirow{2}{*}{$\begin{array}{c}\text { Mean } \\
\text { number of } \\
\text { ray florets / } \\
\text { flower }\end{array}$} & \multirow[t]{2}{*}{$\begin{array}{c}\text { Flower } \\
\text { stalk length } \\
(\mathrm{cm})\end{array}$} & $\begin{array}{l}\text { Flower stalk } \\
\text { thickness }\end{array}$ & \multirow[t]{2}{*}{$\begin{array}{c}\text { No. of } \\
\text { flowers/ } \\
\text { plant }\end{array}$} & $\begin{array}{c}\text { Weight of } \\
\text { flowers/ } \\
\text { plant }\end{array}$ & $\begin{array}{l}\text { Weight of } \\
100 \text { flowers }\end{array}$ & $\begin{array}{c}\text { Yield of } \\
\text { flowers/ plot }\end{array}$ & $\begin{array}{l}\text { Yield of } \\
\text { flowers/ ha }\end{array}$ \\
\hline & $(\mathbf{c m})$ & & & $(\mathbf{m m})$ & & (g) & (g) & (kg) & (tonne) \\
\hline MG-7-3 & 5.85 & 141 & 14.404 & 2.9 & 162.53 & 438.59 & 262 & 8.37 & 23.19 \\
\hline MG-8-1 & 5.69 & 99.3 & 15.75 & 2.9 & 193.27 & 481.27 & 236 & 9.03 & 25 \\
\hline MG-8-2 & 6.1 & 100.7 & 10.45 & 2.9 & 221.1 & 505.81 & 221 & 9.52 & 26.36 \\
\hline MG-9-1 & 6.82 & 148.05 & 15.65 & 2.9 & 242.43 & 631 & 333 & 12.02 & 33.3 \\
\hline MG-9-2 & 5.89 & 145.6 & 13.79 & 3 & 164.51 & 437.06 & 259 & 8.34 & 23.11 \\
\hline MG-9-3 & 5.94 & 145.8 & 15.75 & 2.9 & 172.1 & 438.78 & 249 & 8.38 & 23.2 \\
\hline MG-9-4 & 6.16 & 146.4 & 16.56 & 2.9 & 172.05 & 417.12 & 231 & 7.94 & 22 \\
\hline MG-10-1 & 5.23 & 133.6 & 12.16 & 3 & 167.18 & 361.61 & 206 & 6.84 & 18.93 \\
\hline MG-10-2 & 5.61 & 134.6 & 15.43 & 3 & 212.82 & 505.45 & 224 & 9.51 & 26.34 \\
\hline MG-10-3 & 6.15 & 134.3 & 12.23 & 2.9 & 180.14 & 414.04 & 228 & 7.88 & 21.83 \\
\hline MG-10-4 & 5.59 & 131.8 & 16.93 & 3 & 155.84 & 351.05 & 212 & 6.62 & 18.34 \\
\hline MG-10-5 & 5.21 & 130.8 & 14.33 & 2.9 & 201.09 & 433 & 212 & 8.26 & 22.88 \\
\hline MG-11-1 & 4.61 & 111.2 & 13.51 & 2.9 & 179.85 & 431.74 & 233 & 8.24 & 22.81 \\
\hline MG-12-1 & 4.87 & 142.8 & 12.55 & 2.8 & 171.53 & 445.82 & 259 & 9.02 & 24.98 \\
\hline MG-12-2 & 5.38 & 110.2 & 13.31 & 2.9 & 171.6 & 407.01 & 226 & 7.74 & 21.44 \\
\hline MG-12-3 & 5.45 & 110.6 & 10.7 & 2.8 & 185.05 & 423.61 & 218 & 8.07 & 22.36 \\
\hline MG-13-1 & 5.38 & 118.6 & 13.42 & 3 & 197.85 & 458.61 & 220 & 8.57 & 23.75 \\
\hline MG-14-1 & 5.63 & 140.6 & 11.19 & 2.9 & 204.49 & 390.13 & 190 & 7.41 & 20.51 \\
\hline Local (C) & 5.34 & 140.4 & 11.93 & 2.9 & 226.91 & 488.67 & 202 & 9.18 & 25.41 \\
\hline SEm \pm & 0.11 & 3.39 & 0.79 & 0.15 & 25.35 & 35.05 & 24.25 & 0.7 & 1.95 \\
\hline CD at $5 \%$ & 0.32 & 9.7 & 2.25 & NS & 72.52 & 100.26 & 69.36 & 2.01 & 5.57 \\
\hline
\end{tabular}

Table.3 Qualitative characters

\begin{tabular}{|c|c|c|c|c|}
\hline \multirow[t]{2}{*}{ Genotypes } & \multicolumn{2}{|c|}{ Flower colour } & \multirow{2}{*}{$\begin{array}{c}\text { Shape of } \\
\text { petal }\end{array}$} & \multirow[t]{2}{*}{ Pubescence } \\
\hline & Flower colour & Colour code & & \\
\hline MG-1-3 & Buttercup Yellow & $5 / 1$ & Narrow with pointed tip & Low \\
\hline MG-1-4 & Buttercup Yellow & 5 & Narrow with pointed tip & Low \\
\hline MG-2-1 & Indian Yellow & 6 & Narrow with pointed tip & Medium \\
\hline MG-2-2 & Buttercup Yellow & 5 & Narrow with pointed tip & Low \\
\hline MG-3-1 & Lemon Yellow & $4 / 1$ & Narrow with pointed tip & Medium \\
\hline MG-3-2 & Buttercup Yellow & $5 / 1$ & Narrow with pointed tip & Low \\
\hline MG-3-3 & Indian Yellow & $6 / 1$ & Narrow with pointed tip & Low \\
\hline MG-3-4 & Buttercup Yellow & $5 / 1$ & Narrow with pointed tip & Low \\
\hline MG-4-1 & Indian Yellow & $6 / 1$ & Narrow with pointed tip & Low \\
\hline MG-4-2 & Buttercup Yellow & $5 / 1$ & Narrow with blunt tip & Low \\
\hline MG-4-3 & Aureolin & 3 & Narrow with pointed tip & Low \\
\hline MG-4-4 & Buttercup Yellow & 5 & Narrow with pointed tip & Low \\
\hline MG-5-1 & Lemon Yellow & $4 / 1$ & Narrow with pointed tip & Low \\
\hline MG-5-2 & Indian Yellow & $6 / 1$ & Narrow with pointed tip & Low \\
\hline MG-5-3 & Lemon Yellow & 4 & Narrow with blunt tip & Low \\
\hline MG-5-4 & Buttercup Yellow & 5 & Narrow with pointed tip & Medium \\
\hline MG-5-5 & Indian Yellow & $6 / 1$ & Narrow with pointed tip & Low \\
\hline MG-6-1 & Indian Yellow & 6 & Narrow with pointed tip & Dense \\
\hline MG-6-2 & Lemon Yellow & 4 & Narrow with blunt tip & Low \\
\hline MG-7-1 & Indian Yellow & $6 / 1$ & Narrow with pointed tip & Medium \\
\hline
\end{tabular}




\begin{tabular}{|c|c|c|c|c|}
\hline & \multicolumn{2}{|c|}{ Flower colour } & & \multicolumn{1}{c|}{$\begin{array}{c}\text { Shape of } \\
\text { petal }\end{array}$} \\
\hline MG-7-2 & Flower colour & Colour code & & Now \\
\hline MG-7-3 & Auttercup Yellow & $5 / 1$ & Narrow with blunt tip & Low \\
\hline MG-8-1 & Lemon Yellow & $4 / 1$ & Narrow with pointed tip & Low \\
\hline MG-8-2 & Buttercup Yellow & $5 / 1$ & Narrow with pointed tip & Low \\
\hline MG-9-1 & Indian Yellow & $6 / 1$ & Narrow with pointed tip & Low \\
\hline MG-9-2 & Lemon Yellow & $4 / 1$ & Narrow with pointed tip & Low \\
\hline MG-9-3 & Indian Yellow & $6 / 1$ & Narrow with pointed tip & Low \\
\hline MG-9-4 & Buttercup Yellow & 5 & Narrow with pointed tip & Low \\
\hline MG-10-1 & Lemon Yellow & 4 & Narrow with blunt tip & Low \\
\hline MG-10-2 & Indian Yellow & 6 & Narrow with pointed tip & Medium \\
\hline MG-10-3 & Lemon Yellow & $4 / 1$ & Narrow with pointed tip & Low \\
\hline MG-10-4 & Indian Yellow & $6 / 1$ & Narrow with pointed tip & Low \\
\hline MG-10-5 & Lemon Yellow & 4 & Narrow with blunt tip & Low \\
\hline MG-11-1 & Indian Yellow & $6 / 1$ & Narrow with pointed tip & Low \\
\hline MG-12-1 & Buttercup Yellow & $5 / 1$ & Narrow with pointed tip & Low \\
\hline MG-12-2 & Lemon Yellow & $4 / 1$ & Narrow with pointed tip & Low \\
\hline MG-12-3 & Indian Yellow & $6 / 1$ & Narrow with pointed tip & Low \\
\hline MG-13-1 & Lemon Yellow & $4 / 1$ & Narrow with pointed tip & Low \\
\hline MG-14-1 & Lemon Yellow & 4 & Narrow with pointed tip & Dense \\
\hline Local (C) & Indian Yellow & $6 / 1$ & Narrow with pointed tip & Low \\
\hline
\end{tabular}

Based on flower yield per plot yield per hectare was calculated and treatment differences were significant. The genotype MG-9-1 significantly produced highest flower yield (33.30 t/ha). The minimum flower yield (16.50 t/ha) was recorded by the genotype MG-4-3. These findings are in agreement with Agale (2012) in gailladia and observed that the genotype MG-9 had maximum (36.68 t/ha) flower yield per hectare followed by MG-6 (33.98 t/ha).

The flower yield ranged from 24.20 to 31.37 t/ha. Similarly, Kadam (2014) conducted an experiment on evaluation of different genotypes of marigold (Tagetes erecta L.) under Pune condition and concluded that the variety Double Orange produced maximum flower yield $(21.79 \mathrm{q} / \mathrm{ha})$ which was significantly higher than rest of varieties under study. The minimum yield flower per hectare was recorded (18.27 q/ha). The flower colour was recorded by comparing ray florets with Royal Horticulture Society colour chart. The Aureolin flower colour was observed in MG-4-3 and MG-7-3 genotypes. The Lemon Yellow flower colour was observed in MG-31, MG-5-1, MG-5-3, MG-6-2, MG-8-1, MG9-2, MG-10-1, MG-10-3, MG-10-5, MG-122, MG13-1 and MG-14-1 genotypes. The Buttercup Yellow flower colour was observed in MG-1-3, MG-1-4, MG-2-2, MG-3-2, MG3-4, MG-4-2, MG-4-4, MG-5-4, MG-7-2, MG-8-2, MG-9-4 and MG-12-1genotypes. The Indian Yellow flower colour was observed in MG-2-1, MG-3-3, MG-4-1, MG5-2, MG-5-5, MG-6-1, MG-7-1, MG-9-1, MG-9-3, MG-10-2, MG-10-4, MG-11-1, MG12-3 and local check genotypes. Similar results have been reported by Agale (2012) in gaillardia and observed the colour variation in different genotypes of gaillardia. The data 
revealed that the different genotypes show less variation with respect to their shape of petals. The shape of petals of MG-4-2, MG-53, MG-6-2, MG-7-2, MG-10-1 and MG-10-5 genotypes of gaillardia showed narrow with pointed tip and remaining genotypes showed narrow with blunt tip. In the present investigation, it is revealed that the different genotypes show variation with respect to their pubescence. The genotypes MG-6-1 and MG14-1 had dense pubescence. The genotypes MG-2-1, MG-3-1, MG-5-4, MG-7-1 and MG10-2 had medium pubescence and the remaining genotypes showed comparatively low pubescence (Table 2 and 3 ).

Based on the results obtained from the present one year experimentation, it is concluded that, the genotypes viz., MG-9-1, MG-7-2, MG-62, MG-2-2, MG-5-5 and MG-2-1 showed better growth performance for plant height, spread and branches per plant. All these genotypes showed better performance in respect of flower and yield characters. The straight varieties can be developed from these genotypes in gaillardia. All these genotypes having good quality flower and yield attributes showed promise for further improvement in gaillardia.

\section{References}

Agale, M. G. 2012. Performance of different genotypes of gaillardia (Gaillardia pulchella L.) M. Sc. (Hort.) thesis submitted to Mahatma Phule Krishi Vidyapeeth, Rahuri (Maharashtra).

Bhati, R. A. and Chitkara, S. D. 1989. A note on comparative performance of three cultivars of marigold. Journal of Horticulture Science, 17 (3-4): 204-206.

Bhuyar, A. R., Khiratkar, S. D., Shahakar, A. W., Dharmik, Y. B., Deshmukh, N. A. and Golliwar, V. J. 2004. Growth, quality and yield performance of gerbera under polyhouse condition.
Journal of Soils and Crops. 14 (2): 317319.

Bose, T. K., Yadav, L. P., Pal, P., Das, P. and Parthasarthy, V. 2003. Commercial flowers. $2^{\text {nd }}$ Edition. Naya Udyog, Kolkata. Vol. 2: 852-853.

Carig, R. M. 1977. Proceeding Florida State Horticultural Society, 90: 108-110.

Cox, R. A. and Klett, J. E. 1984. HortScience, 19: 856-858.

Jagtap, H. D. 2013. Evaluation of china aster (Callistephus chinensis (L.) Nees.) genotypes. M.Sc. (Agri.) thesis submitted to Mahatma Phule Krishi Vidyapeeth, Rahuri, (Maharashtra).

Kadam, P. T. 2014. Evaluation of different genotypes of marigold (Tagetes erecta L.). M.Sc. (Agri.) thesis submitted to Mahatma Phule Krishi Vidyapeeth, Rahuri, (Maharashtra).

Kale, V. 2002. Variability, heritability, correlation and path analysis in gaillardia (Gaillardia pulchella). M.Sc. (Agri.) thesis submitted to University Agricultural Sciences, Dharwad (Karnataka).

Mohanty, A., Mohanty, C. R. and Mahapatra, K. C. 2003. Heterosis studies in African marigold. Journal of Ornamental Horticulture, 6 (1): 55-57.

Moringa, Y., Fukushima, E., Kano, T. and Yamasaki, Y. 1929. Botanical Magazine, Tokyo, 43: 589.

Palai, S. K., 2009. Comparative studies on performance of spray chrysanthemum under open and naturally ventilated polyhouse. Journal of Ornamental Horticulture, 12 (2): 138-141.

Palai, S. K., Mohpatra, A., Patnaik, A. K. and Das, P. 1999. Evaluation of spray chrysanthemum for commercial floricultural under Bhubaneswar conditions. Orissa Journal of Horticulture, 27 (1): 34-36.

Panchaude-Mattei, E. 1990. Revue Horticole, 309: 29-31. 
Robinson, M. L. and Schultz, U. E. 1995. Proceeding Florida State Horticultural Society, 107: 194-196.

Swaroop, K., Prasad, K. V. and Raju, D. V. S. 2006. Evaluation of standard chrysanthemum cultivar under low cost polyhouse and open field conditions. Journal of ornamental Horticulture, 9 (1): 69-70.

Swaroop, K., Prasad, K. V. and Raju, D. V. S. 2008. Evaluations of chrysanthemum (Dendranthema grandiflora Tzevlev) germplasm in winter season under Delhi condition Journal of Ornamental Horticulture, 11 (1): 58-61.

Swaroop, K., Saxena, N. K. and Singh, K. P. 2004. Evaluation of China aster varieties under Delhi condition. Journal of Ornamental Horticulture, 7 (1): 127128.

Zosiamliana, J. H., Reddy, G. S. N. and Rymbai, H. 2011. Evaluation of China aster (Callestephus chinensis (L.) ness) cultivars. Journal of Ornamental Horticulture, 14 (3-4): 54-58.

\section{How to cite this article:}

Nagesh Gawade, S.G. Bhalekar, Pushpanjali Bhosale, S.M. Katwate and Vaibhav Wadekar. 2018. Studies on Different Genotypes of Gaillardia (Gaillardia pulchella L.) for Quantitative and Qualitative Performance. Int.J.Curr.Microbiol.App.Sci. 7(03): 1030-1039. doi: https://doi.org/10.20546/ijcmas.2018.703.123 